\title{
Gorlin-Chaudhry-Moss syndrome
}

INSERM

\section{Source}

INSERM. (1999). Orphanet: an online rare disease and orphan drug data base. GorlinChaudhry-Moss syndrome. ORPHA:2095

Gorlin-Chaudhry-Moss (GCM) syndrome is a multiple congenital anomaly syndrome characterized by craniofacial dysostosis, facial dysmorphism, conductive hearing loss, generalized hypertrichosis, and extremity, ocular and dental anomalies. 